\title{
La experiencia de redes de saber y conocimiento en la investigación de maestros y maestras en Cundinamarca
}

\author{
THE EXPERIENCE OF WISDOM AND KNOWLEDGE NETWORKS IN THE RESEARCH OF TEACHERS IN \\ CUNDINAMARCA
}

A EXPERIÊNCIA DE REDES DE SABER E CONHECIMENTO NA PESQUISA DE PROFESSORES E PROFESSORAS EM CUNDINAMARCA

Camilo Andrés Valderrama Alarcón* / cvalderrama@pedagogica.edu.co

Martha Cecilia Cedeño Pérez ${ }^{* *} /$ mccedenop@pedagogica.edu.co

Jorge Enrique Ramírez ${ }^{* * *}$ / jeramirezm@pedagogica.edu.co

\section{Resumen}

En este artículo de reflexión se presentan los resultados de un proyecto de investigación, extensión y proyección social cuyo propósito fundamental fue la construcción de redes de maestras y maestros investigadores de educación básica y media en 109 municipios de Cundinamarca. Dicha experiencia fue llevada a cabo por la Universidad Pedagógica Nacional entre los años 2014 y 2016 en alianza con la Secretaría de Educación de Cundinamarca, la Universidad de los Andes, la Universidad Jorge Tadeo Lozano y la Corporación Universitaria Minuto de Dios. Las redes creadas se configuraron como espacios de formación basados en el intercambio de experiencias pedagógicas situadas desde el saber y conocimiento de los maestros y las maestras, sus anhelos, frustraciones y construcción de alternativas pedagógicas.

\section{Resumo}

Neste artigo de reflexão se apresentam os resultados de um projeto de investigação extensão e projeção social cujo propósito fundamental foi a construção de redes de professoras e professores investigadores de educação básica e media em 109 municípios de Cundinamarca. Essa experiência foi realizada pela Universidade Pedagógica Nacional entre os anos 2014 e 2016 em parceria com a Secretaria de Educação de Cundinamarca, a Universidade de los Andes, a Universidade Jorge Tadeo Lozano e a Corporação Universidade Minuto de Dios. As redes criadas foram configuradas como espaços de formação baseados no intercâmbio de experiências pedagógicas situadas desde o saber e o conhecimento dos professores e as professoras, seus desejos, frustrações e construção de alternativas pedagógicas.
Palabras clave

Redes, ciencia, tecnología, innovación, profesión docente, educación

\section{Keywords}

Networks, science, technology, innovation, teaching profession, education.

\section{Palavras chave}

Redes, ciência, tecnologia, inovação, profissão docente, educação

\footnotetext{
Profesor Universidad Pedagógica Nacional. Magíster en Desarrollo Educativo y Social.

* Profesora Universidad Pedagógica Nacional. Doctora en Antropología del Espacio y el Territorio.

..* Profesor Universidad Pedagógica Nacional. Especialista en Currículo y Pedagogía.
}

Fecha de recepción: 27 de febrero de 2016 / Fecha de aprobación: 01 de mayo de 2016 


\section{Presentación}

Uno de los desafíos iniciales para realizar la investigación, y de lo cual se desprenden objetivos como la creación de redes de maestras y maestros investigadores, fue identificar un concepto de red que tuviese una relación estrecha con la labor pedagógica, debido a que existen múltiples definiciones sobre dicho concepto. En ese sentido, Albornoz y Alfaraz (2006) plantean la existencia de cuatro grandes visiones. La primera de ellas es la sistémica, que concibe la red a partir de un objetivo establecido por una dirección, y al cual todos sus integrantes, ya sean personas o instituciones, se integran de manera funcional para alcanzar la meta previamente establecida; esta perspectiva no se avino a los intereses del proyecto en mención, por cuanto el principio de homogeneidad, del cual parte, no se evidencia entre el cuerpo docente, que es, por antonomasia, un grupo heterogéneo, con diversos modos de ejercer su actividad pedagógica y, por lo tanto, con distintas visiones, intereses y metodologías en su práctica docente cotidiana.

Un segundo enfoque hace énfasis en el uso de las tecnologías de la información y la comunicación (TIC) y su capacidad para la construcción de conocimiento y el manejo de la información, partiendo del hecho de que Internet potencializa la comunicación, permite la confluencia de distintos actores y la circulación de la información de manera eficiente y válida; sin embargo, para el caso de Cundinamarca, es difícil fundamentar la construcción de redes a través de las nuevas tecnologías, debido al precario nivel de conectividad ${ }^{1}$ que se evidencia en las instituciones educativas; sobre todo, en las áreas rurales².

La tercera concepción tiene un claro matiz económico, pues se piensa la red como un mecanismo encaminado

1 El nivel de penetración de internet con el que cuentan los municipios del departamento de Cundinamarca es muy escaso: actualmente oscila entre el $1 \%$ y el $21 \%$. Fuente: MinTIC, en: Vive Digital Colombia (2016).

2 En el informe de 2012 de la Secretaría de la Conectividad de Cundinamarca se muestra que el $56,8 \%$ de los municipios disponen de conectividad a Internet con fibra óptica y que el $100 \%$ de los municipios tienen acceso a Internet en sus principales instituciones públicas. En: SED Cundinamarca (2012). hacia la innovación y la generación de nuevas posibilidades de mercados; esta perspectiva dista también de las condiciones del ejercicio de la docencia, donde la innovación se relaciona con nuevas estrategias pedagógicas en el proceso de enseñanza y aprendizaje, y no con una visión meramente económica.

Una última mirada sobre la noción de red es la relacionada con la suma o "adicionalidad" de capacidades, la cual hace énfasis en una forma organizativa que busca facilitar el trabajo interdisciplinario para producir nuevo conocimiento. Desde este enfoque se articula el proyecto para la construcción de una red de maestras y maestros en el departamento de Cundinamarca, del cual da cuenta esta experiencia.

La constitución de redes es una estrategia que potencializa la ampliación y consolidación de un capital social entre los distintos actores que la conforman, entendiendo dicho capital en el mismo sentido que Bourdieu (1985) "el agregado de los recursos reales o potenciales que se vinculan con la posesión de una red duradera de relaciones más o menos institucionalizadas de conocimiento o reconocimiento mutuo" (p. 248). A través de espacios virtuales y presenciales, los miembros de las redes desarrollaron un conjunto de relaciones de solidaridad que se manifiestan en intercambios materiales o simbólicos reconocidos y valorados por ellos.

Las búsquedas y las acciones de los maestros y las maestras de Cundinamarca por la investigación en la escuela no son recientes: se remontan a las reivindicaciones del Movimiento Pedagógico y a propuestas posteriores como la Expedición Pedagógica Nacional y la Investigación como Estrategia Pedagógica en el Programa Ondas de Colciencias. Para Mejía y Manjarrés (2013), estos desarrollos que tienen como base la movilización social de actores en estructuras de red generan reflexión, acción y participación. En este sentido, la experiencia en la constitución de redes no es un hecho nuevo, pues las redes en sí mismas han sido formas como el magisterio colombiano obtiene encuentros, desencuentros, intercambios y vías para canalizar sus propios esfuerzos y articular los de las instituciones donde desarrollan sus prácticas pedagógicas en el aula y fuera de ella. Para Batista (2004), las redes de profesores pueden 
desempeñar un papel fundamental, por cuanto no se articulan de manera jerarquizada, pues los espacios de reflexión son horizontales y flexibles; en coherencia con ello, Martínez (2008) afirma que las redes no surgen por imposición ni funcionan como un sistema biológico o normativo asociado directamente a intereses políticos, administrativos o institucionales.

\section{Construcción de redes e intercambio de saberes}

Sobre el modo como el cuerpo docente desarrolla su labor pedagógica existe una diversidad de miradas. Para Unda (2002), por ejemplo, existe una permanente

(...) tensión entre posiciones múltiples y variadas, donde coexisten lo nuevo y lo viejo en un mismo maestro, en un mismo centro escolar, nos muestra una escuela viva, atravesada por vectores con distintas orientaciones y magnitudes de fuerza, movida por sus actores, los cuales a su vez tienen distintas maneras de entender y realizar sus prácticas, de entender y realizar los 'cambios'. Se ensayan y se asumen relaciones diferentes con el conocimiento, con los contextos, con el poder; procesos diferenciales de subjetivación y de construcción de lo colectivo. (p.3).

Esa multiplicidad en las formas de encarar el acto educativo entraña diversas dinámicas que no encierran contradicción, sino, más bien, la voluntad de buscar senderos válidos que permitan desarrollar procesos de enseñanza-aprendizaje más contextualizados y significativos. Más allá, estos mismos elementos implican una complejidad a la hora de pensar en la construcción de una red de docentes que trascienda en el tiempo y aúna esfuerzos e intereses; máxime, cuando está enfocada al aspecto investigativo.

Por lo anterior, la construcción de la red de maestras y maestros investigadores se constituyó como un sistema abierto, en el cual se establecieron relaciones multidireccionales y flexibles que permitieron reafirmar su saber pedagógico construido desde lo individual y lo colectivo. Eso significó la asunción de una estrategia de formación que hizo posible el intercambio de saberes y de prácticas pedagógicas, en un esfuerzo por ligar lo grupal y lo particular como una estrategia para potenciar, simultáneamente, los procesos de subjetivación. En este sentido, la red hace posible el fortalecimiento de un conjunto de relaciones y reconocimientos que permite al cuerpo docente reafirmarse en sus estrategias y sus formas singulares de abordar su labor docente, al tiempo que alimenta sus propuestas educativas y da una fuerza colectiva a sus acciones, que de otra forma no trascenderían las fronteras del salón de clase.

Con esa perspectiva, la apuesta en la configuración y la consolidación de redes fue la de crear espacios de trabajo relacionados con aquellas problemáticas propias de los contextos del cuerpo docente $-y$ de estudiantes-, sin aislarlo de su práctica pedagógica ni de la posibilidad de reflexionar sobre ella y sobre los cuestionamientos que esta les plantea. En tal sentido, se puede decir que los intercambios con otros especialistas se establecieron desde la propia experiencia de las maestras y los maestros implicados en el proceso, pero no como una forma de sustituir su propio pensamiento sobre su labor, sino, al contrario, para empoderarlos sobre la pertinencia y la valía de su labor educativa in situ.

\section{Etapas en la construcción de redes de maestros y maestras investigadores}

La configuración y la consolidación de redes de docentes investigadores en los términos ya planteados significaron un desarrollo metodológico que comprendió cuatro fases. La primera de ellas fue la etapa de diseño, en la que se logró la caracterización de la producción pedagógica que adelantaron docentes a lo largo de los últimos dos años en 109 municipios de Cundinamarca; esta identificación fue posible gracias al uso de técnicas como la entrevista, la encuesta, la cartografía social y el análisis documental. En esta fase se reconocieron 540 experiencias pedagógicas en las 15 provincias del departamento, que posteriormente fueron consignadas en una base de datos.

La segunda fase fue la de configuración y motivación, cuyo propósito se encaminó a construir una taxonomía que agrupara las distintas investigaciones pedagógicas identificadas durante la fase anterior —la de diseño-. 
Para motivar la participación del cuerpo docente en las redes, se realizaron convocatorias para presentar proyectos de investigación; dicho llamamiento fue de carácter público, y tenía como requisito que las investigaciones fueran lideradas por este y trabajadas de manera colectiva con estudiantes o con otros docentes. Como resultado de esta iniciativa, se seleccionaron 1.000 proyectos elaborados por profesores y estudiantes y 174 investigaciones pedagógicas adelantadas por grupos de profesores. Todas esas propuestas partieron de problemáticas observadas en los distintos contextos escolares, y fueron financiadas económicamente con recursos del programa de formación en CTel.

La tercera fase correspondió a la de desarrollo y consolidación. En ella, a través del acompañamiento a los grupos de investigación consolidados en las fases anteriores, fue posible establecer un diálogo de manera presencial y virtual con otros docentes investigadores y especialistas e instituciones que desde su experiencia y sus saberes aportaron elementos valiosos para la resignificación de su labor pedagógica y para la articulación de intereses, motivaciones e iniciativas con el ánimo de cualificar su ejercicio docente.

La cuarta y última fase fue la de sostenibilidad y seguimiento, que permitió el surgimiento y el empoderamiento de líderes en las distintas provincias de Cundinamarca, quienes dinamizaron las redes construidas e hicieron nuevas propuestas para su consolidación y su movilización. Esta etapa final aseguró, en cierta medida, el fortalecimiento y la trascendencia de las distintas redes configuradas a lo largo del tiempo que duró el proceso de investigación.

En términos generales, se puede decir que la construcción de redes de maestras y maestros investigadores se fundamentó metodológicamente en tres pilares: el reconocimiento de la investigación como un elemento esencial en la labor docente, lo cual se evidenció en la financiación y el acompañamiento de 1.174 proyectos pedagógicos que fueron diseñados y desarrollados por ellos; el favorecimiento del diálogo de saberes, que tuvo como eje central el desarrollo de las propuestas investigativas cuyos espacios se configuraron a través de dos estrategias: el uso de una plataforma virtual, donde se identificaron 1.485 experiencias pedagógicas y se publicó material relevante de las distintas temáticas abordadas en el curso de formación en investigación, al tiempo que los docentes tenían la oportunidad de participar en foros de discusión y chats; y la realización de eventos académicos donde docentes, estudiantes y especialistas de otras instituciones dialogaron en torno a problemáticas relacionadas con el contexto educativo, socializaron sus avances en los procesos investigativos y las dificultades encontradas en dichos transcursos. El tercer pilar metodológico fue el desarroIlo de dos estrategias para la difusión del conocimiento pedagógico construido a partir de su experiencia investigativa: la participación de docentes en eventos académicos de carácter nacional e internacional y la publicación impresa y digital de los resultados de sus investigaciones.

Como resultado del recorrido enunciado hasta aquí, se pueden mencionar los siguientes hallazgos:

1. En la producción pedagógica investigativa de las maestras y los maestros de Cundinamarca, el equipo de investigación logró identificar siete grandes tendencias que sirvieron como elemento articulador de las redes:

a. Ambiente y biodiversidad: en estas iniciativas de trabajo, el cuerpo docente se enfocó en establecer relaciones entre los ecosistemas y la cultura. Los proyectos presentados reconocen la naturaleza como proveedora de bienes y servicios necesarios para la supervivencia de la sociedad. En este sentido, los intereses de los docentes se enfocaron en reflexionar sobre los impactos ambientales originados por el desarroIlo de actividades humanas, la conservación y el uso sustentable de los recursos, la degradación ambiental y la pérdida de la biodiversidad. Los ejercicios investigativos presentes en esta red fomentan una perspectiva crítica y ética frente a las realidades ambientales de los territorios en los cuales surgieron y se desarrollaron.

b. Agropecuario: quienes hacen parte de esta red relacionan el tema agrícola con los aspectos históricos, sociales y económicos del departamento de Cundinamarca. En ese sentido, los y 
las docentes reflexionan sobre los sistemas y las técnicas de producción, la relación del suelo y el agua con las actividades agropecuarias, los efectos de los procesos productivos en la dinámica territorial y la viabilidad de la implementación de tecnologías agropecuarias novedosas y ancestrales, así como el uso de alternativas ecológicas y biotecnológicas, con una visión analítica y crítica frente a las realidades de los sectores rurales de la región, enfrentados a dinámicas que afectan la vida de los pequeños productores que viven del sustento de la tierra.

c. Creaciones comunicativas y estéticas: en esta red se concentran los proyectos asociados al reconocimiento de lo cultural, lo artístico y lo literario, vistos como múltiples maneras de comprender la realidad. Se configuran como estrategias comunicativas y estéticas que promueven las relaciones con el entorno, al considerar los estilos de vida, el contexto social y las dinámicas específicas establecidas por los grupos humanos.

d. Derechos humanos y democracia: en esta red se reúnen experiencias pedagógicas relacionadas con la cultura política de la escuela, el departamento y el país. Los y las docentes plantean análisis de dinámicas actuales consecuencia de procesos históricos de violencia y resolución de conflictos. La red investiga en el ejercicio de los derechos humanos en el territorio, en ciudadanía y participación escolar, en ética y valores, en formación en sexualidad y en derechos de género, así como en temas relacionados con equidad, diversidad, salud, bienestar y convivencia en la escuela.

e. Emprendimiento e innovación: esta red se centra en la identificación de oportunidades existentes en el entorno, en los ámbitos agrícola y pecuario, así como en el sector manufacturero y en el de artesanías, de manera que promuevan la cultura emprendedora, la gestión empresarial, el desarrollo de la creatividad y la innovación para movilizar el mercado a escala territorial, a partir de la trasformación y la introducción de nuevos productos para el desarrollo y la sostenibilidad de la comunidad. f. Educación y pedagogía: esta es la red más grande de todas las que fueron identificadas en el proyecto. El trabajo realizado por los docentes que hacen parte de ella atañe a la formación de sujetos, para que, a partir de una relación dialógica entre profesores y estudiantes, se logre comprender de manera crítica las realidades del entorno, mediante la investigación como alternativa para la producción colectiva del conocimiento. Ello ha implicado la participación de docentes de distintas disciplinas para lograr un mayor alcance y la solución a problemáticas existentes en los territorios.

g. Tecnología: aquí se abordan problemas investigativos enfocados en las nuevas tecnologías en relación con diferentes áreas del saber. Se presentan análisis de las experiencias de ambientes de aprendizaje mediados por TIC y apoyados en acciones metodológicas basadas en la creatividad. Se plantearon proyectos en producción informática, diseño y creación de objetos; también, en sistematización (programación en robótica) en manejo del agro, en optimización de los recursos naturales, en temáticas ambientales sobre biodiversidad y en la transformación de objetos funcionales a través del reciclaje y los procesos informáticos.

En la tabla 1 se muestra el número de proyectos asociados a cada una de las redes mencionadas:

Tabla 1. Proyectos y redes

\begin{tabular}{|c|c|}
\hline Red temática & $\begin{array}{c}\text { Número de proyectos } \\
\text { identificados }\end{array}$ \\
\hline Educación y pedagogía & 394 \\
\hline Ambiente y biodiversidad & 357 \\
\hline $\begin{array}{c}\text { Derechos humanos y } \\
\text { democracia }\end{array}$ & 185 \\
\hline $\begin{array}{c}\text { Agropecuario } \\
\text { estéticas }\end{array}$ & 184 \\
\hline $\begin{array}{c}\text { Creaciones y comunicaciones } \\
\text { Emprendimiento }\end{array}$ & 180 \\
\hline Tecnología & 90 \\
\hline
\end{tabular}

Fuente: elaboración propia 
En lo que respecta a la consolidación de redes de maestras y maestros investigadores a través de las estrategias de socialización, la participación en eventos internacionales y la publicación impresa y digital de los artículos de investigación, se obtuvieron los siguientes resultados (tabla 2 ):

\section{Tabla 2. Resultados de participación en eventos internacionales}

\begin{tabular}{|c|c|}
\hline Cobertura & Cantidad \\
\hline $\begin{array}{c}\text { Docentes participantes en eventos } \\
\text { nacionales e internacionales }\end{array}$ & 95 \\
\hline Provincias & 12 \\
\hline Municipios & 39 \\
\hline $\begin{array}{c}\text { Instituciones Educativas Departamentales } \\
\text { (IED) }\end{array}$ & 76 \\
\hline $\begin{array}{c}\text { Países: México (24), Chile (26), Argentina } \\
\text { (6), Venezuela (25), Perú (2), Ecuador } \\
\text { (2), Bolivia (3), Uruguay (2) y } \\
\text { Colombia (5) }\end{array}$ & 9 \\
\hline $\begin{array}{c}\text { Ciudades nacionales e internacionales } \\
\text { Áreas temáticas abordadas: } \\
\text { Educación y pedagogía (35), Ambiente y } \\
\text { biodiversidad (13), Tecnología (12), } \\
\text { Emprendimiento (11), Creaciones } \\
\text { comunicativas y estéticas (10), } \\
\text { Derechos humanos y democracia (8), } \\
\text { Agropecuaria (6) }\end{array}$ & 22 \\
\hline
\end{tabular}

Fuente: elaboración propia

2. La estrategia de difusión de las experiencias investigativas de docentes a través de la publicación de material impreso ha tenido como resultado, hasta la fecha, la creación de seis libros: tres publicados por la Universidad Pedagógica Nacional; dos, por Uniminuto, y uno, por la Universidad de los Andes. Todo esto es evidencia de la capacidad investigativa de los docentes del departamento.

3. Las siete redes temáticas se dinamizaron mediante el intercambio de bienes materiales y simbólicos, el cual propició nuevos abordajes en el trabajo de aula y la consolidación de un conjunto de relaciones de solidaridad entre los distintos actores educativos. Las redes se con- virtieron así en una estrategia para el desarrollo de capital social entre los docentes de Cundinamarca, permitiendo concluir que "la existencia de un conjunto de relaciones sociales no es algo natural, sino que es el resultado de una serie de estrategias colectivas, creadas de manera consciente o inconsciente, que son útiles a corto o a largo plazo" (Valderrama, 2013, p.78).

\section{Conclusiones y recomendaciones}

Los procesos desarrollados a lo largo del proyecto permitieron la definición de siete tendencias temáticas relacionadas con la labor pedagógica que adelantan los docentes en Cundinamarca, a saber: ambiente y biodiversidad; agropecuaria; creaciones comunicativas y estéticas; derechos humanos y democracia; emprendimiento; educación, y tecnología.

Es posible construir una red de maestros y maestras investigadores teniendo como eje central sus intereses y reconociendo su labor pedagógica mediante el desarrollo de estrategias de difusión de su saber pedagógico, tales como la participación en eventos internacionales y la publicación de sus hallazgos investigativos.

El trabajo en red con docentes no se puede fundamentar exclusivamente en las TIC, pues en el departamento de Cundinamarca existen problemas de conectividad que limitan el uso de dicho recurso; por tal razón, es necesario hacer un trabajo complementario con encuentros presenciales, donde los maestros y maestras tengan la posibilidad de compartir sus conocimientos y sus experiencias reconociéndose como un colectivo productor de saber pedagógico.

El trabajo en red es una estrategia de formación entre iguales que potencializa la construcción colectiva de un saber, sin desconocer las individualidades de sus integrantes. Por ello, es fundamental que los docentes trabajen de manera articulada, pues las estructuras burocráticas construidas en torno a la escuela superan cualquier esfuerzo individual y la red es una posibilidad de enlazamiento entre diferentes sectores y organizaciones que pueden llegar a transformar la escuela, y, por eso mismo, el entorno en el cual está inmersa. 


\section{Referencias}

Albornoz, M. y Alfaraz, C. (2006). Redes de conocimiento: construcción, dinámica y gestión. Buenos Aires: Centro de Estudios sobre Ciencia, Desarrollo y Educación Superior.

Batista, J. (2004). A rede de investigacao na escola. Uma experiencia em gestacao. Revista Nodos y Nudos 2 (16), 69-78.

Bourdieu, P. (1985). The Forms of Social Capital En J. Richardson (ed). Handbook of Theory and Research for the Sociology of Education. (pp. 241-258). New York: Greenwood.

Martínez, M. (2008). Redes pedagógicas: la constitución del maestro como sujeto político. Bogotá: Magisterio.

Mejía, M. y Manjarrés, M. (2013). La investigación como estrategia pedagógica, una propuesta desde el sur. Bogotá: Desde Abajo.
SED Cundinamarca. (2012). Informe de gestión 2012. Secretaría de Conectividad. Recuperado de: http://www.cundinamarca.gov.co/wps/wcm/connect/f0f72 837-87c6-42d6853a-a0b5a6037ac2/INFORME+GESTION+SECRETARIA+C ONECTIVIDAD+Feb2012.pdf?MOD=AJPERES\&Informe $\% 20$ de\%20gesti\%C3\%B3n\%2006

Unda, M.P. (2002).The experience of the teaching expedition and teacher networks. Prospects XXXII, 3, 333-345, Unesco.

MinTIC. Vive Digital Colombia. (2016). Estrategia TIC Colombia. Recuperado de: http://estrategiaticolombia.co/estadisticas/stats. php id $=25 \&$ pres $=$ det $\&$ jer $=2 \& \operatorname{cod}=25$

Valderrama, C. (2013). Conociendo reglas e inventando estrategias. Bogotá: Fundación Centro Internacional de Educación y Desarrollo Humano.

\section{Diálogo del conocimiento}

Comparto algunos comentarios acerca de las ideas, prácticas y propuestas desarrolladas por los docentes Camilo Valderrama, Martha Cecilia Cedeño y Jorge Enrique Ramírez en su artículo "La experiencia de redes de saber y conocimiento en la investigación de maestros y maestras en Cundinamarca".

En principio una idea central: el artículo se inscribe y cobra sentido histórico - contextual, en el marco de decisiones y acciones relativas a los significados, a los valores que representan, las responsabilidades y compromisos diversos que se asignan a los maestros en la escuela y fuera de ella. Este proyecto de investigación y de extensión que los autores describen corresponde a un ejercicio esencial de naturaleza ética y política fundamentalmente, en cuanto se orientó hacia la dignificación de la labor de los educadores y por ende hacia la permanente resignificación y deconstrucción, de los maestros, los niños y jóvenes, la escuela y la educación en general.

Llama la atención, la noción de "red" fundamentada en la idea de "adicionalidad" que los autores emplearon para fundamentar y generar los eventos de "resignificación" antes señalados. Dos aclaraciones importantes son esbozadas en el artículo: en primer lugar, las redes han sido formas históricas del magisterio en Colombia que se dan y evolucionan en torno a encuentros, desencuentros e intercambios. En segundo lugar, la noción de "adicionalidad" se define en su significado general como "la suma de capacidades que facilita el trabajo interdisciplinario y apunta hacia la producción de nuevo conocimiento".

Algunas de las líneas de acción y de logro significativo que, a juicio de quien escribe, se alcanzaron con el desarrollo de este proyecto, son las siguientes:

- El reconocimiento y asignación de sentido a la experiencia de los maestros.

- La consolidación del diálogo y la construcción de relatos significativos.

- El intercambio y la interacción entre los sujetos y las comunidades en torno de la investigación.

- La reconstitución y recreación de los sujetos y las comunidades.

- La reinvención de la escuela.

Temas de la red / La experiencia de redes de saber y conocimiento en la investigación de maestros y maestras en Cundinamarca / Camilo Andrés Valderrama Alarcón / Martha Cecilia Cedeño Pérez / Jorge Enrique Ramírez 
Se resalta lo anterior en cuanto al proceso de formación y de investigación; según se colige de las ideas plasmadas en el artículo, se vinculó a las historias particulares y generales de los profesores en interacción con los distintos actores de la comunidad. Esto se logró mediante la disposición de los distintos actores, principalmente los maestros, en función de la identificación y reconstrucción de sus experiencias, como ya se afirmó. Implicó apelar a la palabra de cada sujeto, de cada grupo o colectivo y desde allí, generar las dinámicas propias del diálogo, intercambio e interacción; además, se construyeron los relatos y los textos que dan cuenta de las experiencias de investigación. De este proceso, y como resultado, emergen tres logros esenciales: la transformación de los sujetos y el cambio en la institución escolar y, por ende, la transformación de la comunidad, tanto en sus rasgos centrales como en sus dinámicas y estructura.

En suma, se trata de un conjunto de propuestas que vale la pena revisar y estudiar, con opciones de trasladar los productos y conclusiones logrados a otros contextos tanto geográficos como temporales.

Nelson Otálora Porras 